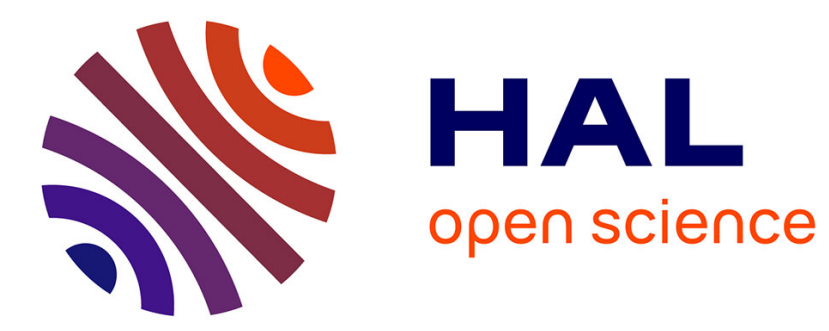

\title{
SMOS images restoration from L1A data: A sparsity-based variational approach
}

J. Preciozzi, Pablo Musé, Andrés Almansa, Sylvain Durand, Ali Khazaal, Bernard Rougé

\section{- To cite this version:}

J. Preciozzi, Pablo Musé, Andrés Almansa, Sylvain Durand, Ali Khazaal, et al.. SMOS images restoration from L1A data: A sparsity-based variational approach. IEEE Geoscience and Remote Sensing Symposium, Jul 2014, Quebec, Canada. pp.2487-2490, 10.1109/IGARSS.2014.6946977 . hal01265931

\section{HAL Id: hal-01265931 https://hal-imt.archives-ouvertes.fr/hal-01265931}

Submitted on 1 Feb 2016

HAL is a multi-disciplinary open access archive for the deposit and dissemination of scientific research documents, whether they are published or not. The documents may come from teaching and research institutions in France or abroad, or from public or private research centers.
L'archive ouverte pluridisciplinaire HAL, est destinée au dépôt et à la diffusion de documents scientifiques de niveau recherche, publiés ou non, émanant des établissements d'enseignement et de recherche français ou étrangers, des laboratoires publics ou privés. 


\section{SMOS IMAGES RESTORATION FROM L1A DATA: A SPARSITY-BASED VARIATIONAL APPROACH}

\author{
J. Preciozzi, P. Musé \\ Univ. de la República, Uruguay
}

\author{
A. Almansa ${ }^{\dagger}$, S. Durand ${ }^{\ddagger}$ \\ ${ }^{\dagger}$ LTCI - Telecom ParisTech \\ $\ddagger$ MAP5 - Univ. René Descartes
}

\author{
A. Khazaal, B. Rougé \\ CESBIO \\ 31400 Toulouse, France
}

\begin{abstract}
Data degradation by radio frequency interferences (RFI) is one of the major challenges that SMOS and other interferometers radiometers missions have to face. Although a great number of the illegal emitters were turned off since the mission was launched, not all of the sources were completely removed. Moreover, the data obtained previously is already corrupted by these RFI. Thus, the recovery of brightness temperature from corrupted data by image restoration techniques is of major interest. In this work we propose a variational approach to recover a super-resolved, denoised brightness temperature map based on two spatial components: an image $u$ that models the brightness temperature and an image $o$ modeling the RFI. The approach is totally new to our knowledge, in the sense that it is directly and exclusively based on the visibilities (L1a data), and thus can also be considered as an alternative to other brightness temperature recovery methods.
\end{abstract}

Index Terms - SMOS, MIRAS, RFI, non-differentiable convex optimization, total variation minimization.

\section{INTRODUCTION}

Meteorological and climate predictions are very sensitive to variables such as surface soil moisture (SSM) and sea surface salinity (SSS). The MIRAS instrument (Microwave Imaging Radiometer by Aperture Synthesis), carried on the SMOS satellite, is capable of measuring the corresponding brightness temperature of both SSM and SSS indirectly, in the Lband microwave, using interferometry and sensing the socalled visibility function [1], defined as the complex crosscorrelation between the two signals collected by each pair $\left(A_{k}, A_{l}\right)$ of antennas:

$V_{k, l}=\frac{1}{\sqrt{\Delta_{k} \Delta_{l}}} \iint_{\|\boldsymbol{\xi}\| \leq 1} U_{k}(\boldsymbol{\xi}) U_{l}^{*}(\boldsymbol{\xi})\left(T_{b}(\boldsymbol{\xi})-T_{r}\right) \tilde{r}_{k l}(t) \frac{e^{-i 2 \pi \mathbf{u}_{k l}^{T} \boldsymbol{\xi}} d \boldsymbol{\xi}}{\sqrt{1-\|\boldsymbol{\xi}\|^{2}}}$

Here, $\mathbf{u}_{k l}$ is the frequency baseline associated to $A_{k}$ and $A_{l}$; $U_{k}, U_{l}$ are the corresponding normalized voltage patterns and $\Delta_{k}, \Delta_{l}$ are the solid angles of the corresponding antennas. The Cartesian coordinates $\boldsymbol{\xi}=\left(\xi_{1}, \xi_{2}\right)$ are the spatial do- main coordinates, restricted to the unit circle. $T_{r}$ is the physical temperature of the receivers (assumed the same for all receivers); $\tilde{r}_{k l}$ is the Fringe-Wash function, a function of the spatial delay $t=\frac{\mathbf{u}_{\mathbf{k} 1}^{T} \boldsymbol{\xi}}{f_{0}}$, where $f_{0}=\frac{c}{\lambda_{0}}$ is the central frequency of observation. Note that the brightness temperature $T_{b}$ is a $2 \mathrm{D}$ function restricted to the unit circle $(\|\boldsymbol{\xi}\| \leq 1)$.

The MIRAS instrument is composed of three arms on a Yshaped configuration, each arm carrying an array of antennas. This configuration leads to a hexagonal sampling grid of the visibility function. Figure 1 shows the star shaped domain $\Omega$ obtained from the MIRAS configuration.

If we denote by $T=T_{b}-T_{r}$, the samples of $T$ in the hexagonal grid could be obtained from the visibility samples by solving the linear system $\mathbf{G} T=V$, where matrix $\mathbf{G}$ represents the discrete linear operator given by (1). Of course, this inverse problem is ill-posed since $\mathbf{G}$ is not invertible (due to the lack of information beyond $\Omega$ ). Hence, additional constraints must be added to the model. In [2], Anterrieu proposes to solve it as a constrained least square problem, imposing that $T$ has no frequency components outside $\Omega$. This problem can be formulated as the unconstrained minimisation of $\left|V-\mathbf{G F}^{*} \mathbf{Z}_{\boldsymbol{\Omega}} \hat{T}\right|_{2}^{2}$ on $\hat{T}$, where $\mathbf{F}^{*}$ denotes the hexagonal Inverse Fourier Transform, $\mathbf{Z}_{\Omega}$ the zero padding operator and $\hat{T}$ the Fourier coefficients of $T$ for frequencies in $\Omega$. Let $\mathbf{J}=\mathbf{G F}^{*} \mathbf{Z}_{\boldsymbol{\Omega}}$, then $\hat{T}=\mathbf{J}^{+} V$ where $\mathbf{J}^{+}$is the pseudo-inverse of $\mathbf{J}: \mathbf{J}^{+}=\left(\mathbf{J}^{*} \mathbf{J}\right)^{-1} \mathbf{J}^{*}$. This is the way the L1a product is obtained, and corresponds exactly to $\hat{T}$. In what follows we will note L1b data product as $D_{L 1 b}$.

Using L1b data, $T$ can be recovered from $D_{L 1 b}$ very easily: $T=\mathbf{F}^{*} \mathbf{Z}_{\Omega} D_{L 1 b}$. Naturally, as we have shown in [3], this simple Fourier inversion leads to potentially very strong Gibbs effects which are partially alleviated (as proposed by [2]) by the use of a Blackman window $\mathbf{B}: T=\mathbf{F}^{*} \mathbf{B Z}_{\Omega} D_{L 1 b}$. As we pointed out in [3], such linear approaches are not well

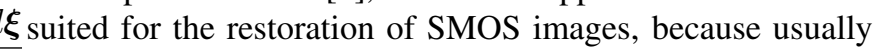
the measurements are polluted by a number of strong outliers that correspond to signals emitted in the L-Band by illegal antennas ${ }^{1}$. Because these outliers have frequencies beyond $\Omega$, very strong Gibbs effects can be seen on the final brightness

\footnotetext{
${ }^{1}$ International radio regulations reserve the L-band is exclusively to the Earth Exploration Satellite Service, space research and radio astronomy.
} 
temperature images (see Figure 2). To eliminate them and to obtain a super-resolved solution, in [3] we proposed to solve the following variational formulation

$$
\min _{u, o}\{\operatorname{TV}(u)+\mu S(o)\} \text { s.t. }\left\|W\left(F(o+u)-D_{L 1 b}\right)\right\|_{2}^{2} \leq|\Omega| \sigma^{2}
$$

where $T V(u)$ denotes the total variation semi-norm of the target image $u, S(o)$ a sparsity measure on the outliers (in practice $\ell_{0}$ or $\ell_{1}$ norms), $W$ is a weighting matrix and $\sigma^{2}$ is the instrumental noise, assumed to be white and Gaussian (see [3] for details). Note that this method allows to automatically separate the restored image $u$ from the outliers. Although this approach produced significantly better results than previous ones (Blackman and direct Fourier inversion), the model is not totally accurate since the restoration is performed starting from the $D_{L 1 b}$, which has already been subject to regularisation.

In this work, we improve our previous model, by directly dealing with visibilities (SMOS L1a data product). The goals are exactly the same than those of our previous work: to detect and remove signal effects generated from illegal emitters (outliers), while at the same time extrapolating the image spectrum in order to minimize Gibbs effects. As we will show in the following sections, this simple data change is not easy to implement because of the lack of regularization in the L1a data, and because of several issues that have to be considered to make the inverse problem numerically tractable. To our knowledge, this is the first work that tackles the problem directly and exclusively from the L1a product. Other approaches, such as [4], first localise the potential outliers based on the L1b product, then simulate the contribution on visibilities of each outlier to remove it from the original L1a data; finally they convert the outlier-free L1a data to L1b (by means of the pseudo-inverse matrix $\mathbf{J}^{+}$) as an intermediate step to recover the temperatures.
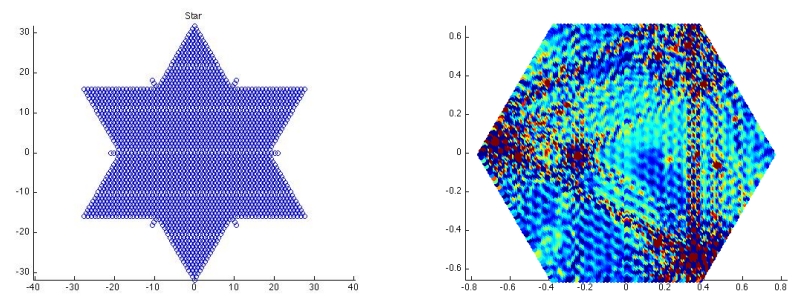

Fig. 1. Spectral domain $\Omega$ as- Fig. 2. Temperature obtained sociated to MIRAS Y-shaped by the inverse Fourier transinstrument. form of the L1b product.

\section{PROPOSED APPROACH}

As we already mentioned, visibilities and bright temperatures are related by the discrete linear operator given by (1). In matrix notation ${ }^{2}$, this is $\mathbf{G} u=V$. As before, the goal is to obtain the temperatures image $u$ from the given visibilities $V$, knowing the non-invertibility of $\mathbf{G}$.

\subsection{Variational formulation}

We propose to recover $u$ by solving the following constrained optimization problem:

$$
\min _{u, o}\{\operatorname{TV}(u)+\mu S(o)\} \text { s.t. }\|\mathbf{G}(o+u)-V\|_{2}^{2} \leq|\Omega| \sigma^{2},
$$

that can be reformulated as an unconstrained problem (see [3] for the details):

$$
\min _{u, o}\left\{\|\mathbf{G}(o+u)-V\|_{2}^{2}+\lambda(\operatorname{TV}(u)+\mu S(o))\right\} .
$$

The first term is the data fidelity term, which acts directly on the visibilities (L1a product). The second term acts as a regularizer and is the same as the one in our previous model; it is designed to separate the outliers $o$ from the brightness temperature map $u$. $T V(u)$ intends to super-resolve $u$ beyond spectral support $\Omega$ while avoiding Gibbs oscillations. The second term seeks to promote a sparse solution to the image of outliers by applying some sparsity operator to $o$. The parameter $\mu$ controls the trade-off between both terms; its choice can be formally derived from geometric considerations on the outliers. For the sake of brevity, we refer the reader to [3].

In order to reduce the "staircasing" effect inherent to many TV minimization methods, we make use of the Spectral $T V$ introduced by Moisan [5] (see [3] for the implementation details). The final method can be stated as follows:

$$
\min _{u, o}\{\underbrace{\frac{1}{2}\|\mathbf{G}(o+u)-V\|_{2}^{2}}_{E_{1}(u, o)}+\underbrace{\lambda\left(\operatorname{TV}_{\mathcal{H}}(u)+\mu S(o)\right)}_{E_{2}(u, o)}\},
$$

where $\operatorname{TV}_{\mathcal{H}}(u)$ denotes the Spectral TV.

\subsection{Numerical implementation}

Numerical minimization is based on a Forward-Backward splitting algorithm [6]. The $k$-th iteration starting from seed $x^{0}=\left(u^{0}, o^{0}\right)$ is

$$
\left\{\begin{array}{rlr}
x^{k+1 / 2} & =x^{k}-\gamma \nabla E_{1}\left(x^{k}\right) \\
x^{k+1} & =\operatorname{prox}_{\gamma E_{2}}\left(x^{k+1 / 2}\right) .
\end{array}\right.
$$

In order to ensure convergence to the minimizer, $\gamma$ must be smaller than $2 / L$, where $L$ is the Lipschitz constant of $\nabla E_{1}$ (in our case $\gamma<689$ ). Because the formulation only changes from the previous one on the data term $E_{1}$, the proximal operator for $E_{2}$ remains the same as in the case of L1b data [3]:

$$
\operatorname{prox}_{\gamma E_{2}}(u, o)=\left(\operatorname{prox}_{\gamma \lambda \mathrm{TV}_{\mathcal{H}}}(u), \operatorname{prox}_{\gamma \lambda \mu\|\cdot\|_{1}}(o)\right) .
$$

\footnotetext{
${ }^{2}$ For the sake of simplicity, we use the same symbol to refer to an image and its vectored form. Disambiguation follows easily from the context.
} 
Now we differentiate $E_{1}$ and we obtain:

$$
\nabla E_{1}(u, o)=\left(\mathbf{G}^{*} \mathbf{G}(u+o)-V, \mathbf{G}^{*} \mathbf{G}(u+o)-V\right) .
$$

$\mathbf{G}^{*} \mathbf{G}$ is a huge full matrix $(16384 \times 16384$ since $\mathbf{G}$ is a $4695 \times 16384$ matrix). Explicit multiplication by this matrix at each iteration of the algorithm is computationally intractable. However, a change of basis to the Fourier domain changes the gradient term to

$$
\nabla E_{1}(u, o)=F^{*}\left(\left(\mathbf{G F}^{*}\right)^{*} \mathbf{G F}^{*} F(u+o)-\left(\mathbf{G F}^{*}\right)^{*} V\right)
$$

which reveals an even larger $(32768 \times 32768)$ but highly sparse matrix $\mathbf{F G}^{*} \mathbf{G F}^{*}$ : to keep the energy at $99.99 \%$, we only need to keep $0.0008 \%$ of the coefficients.

Finally, each iteration for the first step $\left(S(o)=\|o\|_{1}\right)$ can be expressed as follows:

$$
\left\{\begin{array}{l}
u^{k+1 / 2}=u^{k}-\gamma F^{*}\left(\mathbf{F G}^{*} \mathbf{G F}^{*} F(u+o)-\mathbf{F G}^{*} V\right. \\
o^{k+1 / 2}=o^{k}-\gamma F^{*}\left(\mathbf{F G} \mathbf{G F}^{*} F(u+o)-\mathbf{F G}^{*} V\right. \\
u^{k+1}=\operatorname{prox}_{\gamma \lambda \mathrm{TV}}\left(u^{k+1 / 2}\right) \\
o^{k+1}=s_{\gamma \lambda \mu}\left(o^{k+1 / 2}\right) .
\end{array}\right.
$$

This iteration converges to a global minimum, that corresponds to the solution of problem (5) with sparsity operator $S(o)=\|o\|_{1}$. We use this soultion as an initialisation for the second step, where the sparsity operator is non-convex, namely $S(o)=\|o\|_{0}$. For this problem, the same ForwardBackward method can be considered and is guaranteed to converge to a local minimizer [7]. Now, instead of the soft thresholding, the proximal operator for $S(o)$, which is now $\|o\|_{0}$, becomes the hard thresholding $h_{\sqrt{2 \gamma \lambda \mu}}$.

\section{EXPERIMENTS}

To highlight the benefits of the proposed framework, we present two kinds of experiments. In the first one, we compare results from our approach to those obtained by previous works: a Fourier inversion of the L1b data, a simple Blackman apodization to smooth the outliers effects, and our previous L1b minimisation framework presented on [3]. Experiments are run on several snapshots from the SMOS dataset of march 2010. We have set $\sigma$ equal to $5 K$, which is the measurement error reported by the SMOS mission. Figures 4 and 5 show the results obtained for snapshots 996 and 1050. For geographic reference, figure 3 shows how these regions look in Google Earth from approximately the same viewing angles as the SMOS acquisitions.

Note that the acquired images are corrupted by several outliers that considerably degrade the data. It is clear that the present method outperforms both the direct inverse Fourier transform and the Blackman apodization. It also improves the results from our previous work based on L1b data, which resulted in more regularised images and thus, valuable details where lost. It is worth mentioning that the comparison of the results was confirmed by environmental scientists.
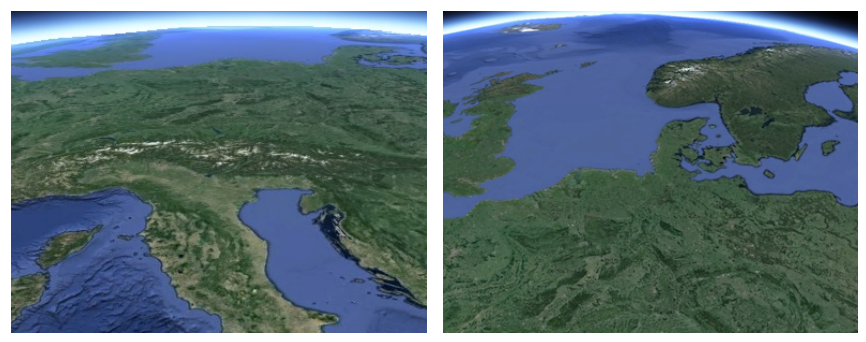

Fig. 3. Google Earth view of two of the regions used on the experiments. The left one corresponds to snapshot 996 and the right one to snapshot 1050 .

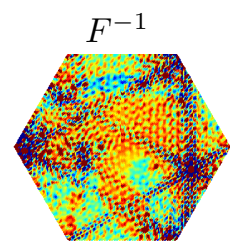

Using L1b method

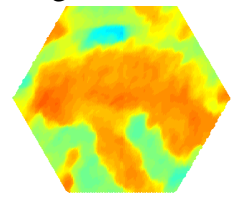

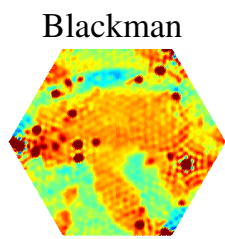

The proposed method

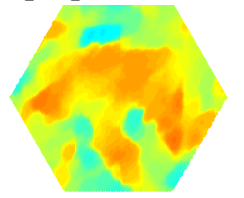

Fig. 4. Comparison between previous works and our method. This snapshot corresponds to Central Europe, with Italy clearly visible, and was taken on march 2010. Color scale was mapped between 0 and 300 Kelvin..

In the second experiment we perform a quantitative evaluation of our method, for which we need a ground truth data to compare with. We generated a simulated set in the following way. We first applied our algorithm to a real acquired image free of outliers. This leads to a denoised image $u_{g t}$ that we selected as ground truth, and then we obtained from it the corresponding visibilities $V_{g t}=\mathbf{G} u_{g t}$. We then generated the outliers image by randomly selecting several pixels, and randomly generating intensity values between 300 and 30000 $\mathrm{K}$. Similarly, this image $T_{\delta}$ was transformed into visibilities: $V_{\delta}=\mathbf{G} T_{\delta}$. Finally, we added noise that follows the given normal noise model with mean 0 and variance $\sigma_{v}$. The final simulated data was then $V_{f}=V_{g t}+V_{\delta}+n_{v}$, which follows exactly our image generation model. Figure 6 shows the results obtained on the simulated dataset. The table shows the estimation error on $u$ for different error norms using both our previous method on L1b data, and the current one based on L1a. These errors were computed over the whole Fourier domain, since in the case of simulated images, no aliasing occurs. In all cases, the standard deviation of the residual measured in the spatial domain was $5 \pm 0.2$, which is in agreement with the expected temperature noise model $\left(\sigma_{T}=5.0\right)$. We can see from this table a consistent improvement on the use 


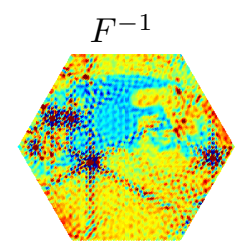

Using L1b method

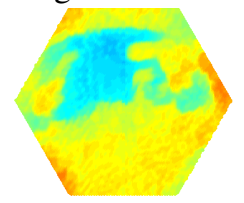

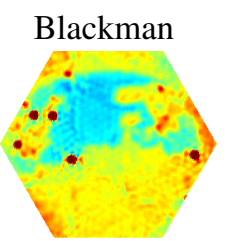

The proposed method

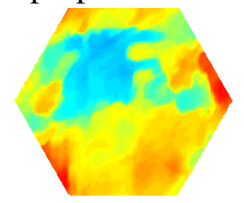

Fig. 5. Comparison between previous works and our method. This snapshot corresponds to North Europe and was taken on march 2010. Color scale were mapped between 0 and 300 Kelvin.

of the proposed method compared with the previous one.

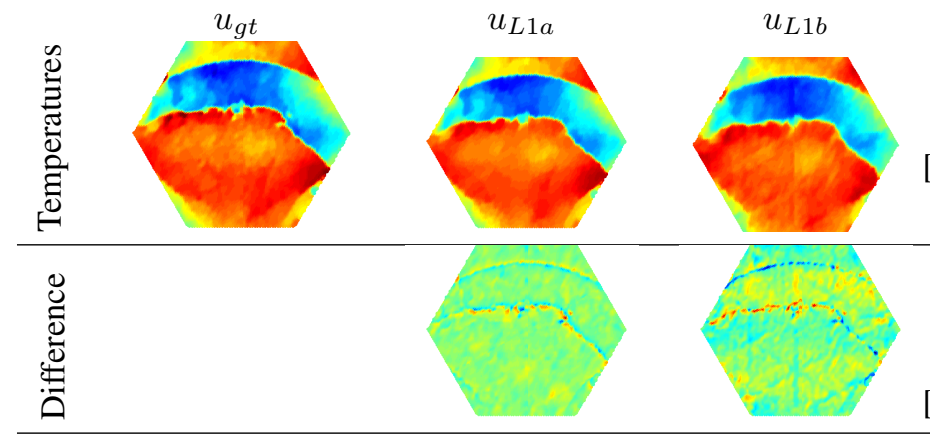

\begin{tabular}{|l|l|l|l|}
\hline & $L_{1}$ & $L_{2}$ & $L_{\infty}$ \\
\hline$u_{L 1 a}-u_{g t}$ & 3.197598 & 5.418558 & 57.679203 \\
\hline$u_{L 1 b}-u_{g t}$ & 9.587280 & 12.994680 & 87.467700 \\
\hline
\end{tabular}

Fig. 6. Results from simulated data. The error is measured over all the image, not only the free of aliasing (FOA) zone.

\section{CONCLUSIONS}

In this article we present a new method to restore SMOS images directly and exclusively from the L1a product. Although the main objetive of the method is to detect and remove the outliers produced by RFI, it can be considered as a new approach to obtain brightness temperature from the visibilities, even in the absence of outliers. The method follows the same methodology of our previous work [3], but adapted to the use of L1a instead of L1b data. We kept the previous idea of using two variables $u$ and $o$ to model brightness temperatures and outliers images separately, but the data term was adapted to use visibilities directly. This modification requires several changes: working directly with visibilities is hard since matrix $\mathbf{G}$ is numerically difficult to invert. We propose then to work on the Fourier domain, which turns the problem more stable with a new matrix $\mathbf{G F}^{*}$ that becomes extremely sparse. This new approach is much closer to the real SMOS image formation model. This is not only a better model from a theoretical point of view: it gives better results because outliers are removed before any regularisation is done (in contrast with L1b methods were the RFI contributions are distributed all along the data). Consequently, the obtained images are less regularised when compared to L1b based methods, and small variations are kept, which is important since each pixel has a resolution between 30 and $50 \mathrm{~km}$.

\section{REFERENCES}

[1] I. Corbella, N. Duffo, M. Vall-llossera, A. Camps, and F. Torres, "The visibility function in interferometric aperture synthesis radiometry," IEEE Trans. Geosci. Remote Sensing, vol. 42, no. 8, pp. 1677-1682, 2004.

[2] E. Anterrieu, "A resolving matrix approach for synthetic aperture imaging radiometers," IEEE Transactions on Geoscience and Remote Sensing, vol. 42, no. 8, pp. 1649 - 1656, aug. 2004.

[3] J. Preciozzi, P. Muse, A. Almansa, S. Durand, F. Cabot, Y. Kerr, A. Khazaal, and B. Rouge, "Sparsity-based restoration of smos images in the presence of outliers," in IEEE International Geoscience and Remote Sensing Symposium 2012, 2012, pp. 3501-3504.

[4] Rita Castro, Antonio Gutiérrez, and José Barbosa, "Iterative thresholding for sparse approximations," IEEE Transactions on geoscience and remote sensing, vol. 50, no. 5, pp. 1440-1447, May 2012.

[5] L. Moisan, "How to discretize the total variation of an image?," ICIAM07 Minisymposia, 2007.

[6] P. L. Combettes and V. R. Wajs, "Signal recovery by proximal forward-backward splitting," SIAM Journal on Multiscale Modeling and Simulation, vol. 4, no. 4, pp. 1168-1200, 2005.

[7] Thomas Blumensath and Mike E Davies, "Iterative thresholding for sparse approximations," The Journal of Fourier Analysis and Applications, vol. 62, pp. 291-294, January 2008. 